\title{
The First Turkish Case of Hypoparathyroidism, Deafness and Renal Dysplasia (HDR) Syndrome
}

\author{
Hakan Döneray1, Takeshi Usui2, Avni Kaya1, Ayşe Sena Dönmez3 \\ ${ }^{1}$ Atatürk University Faculty of Medicine, Department of Pediatric Endocrinology, Erzurum, Turkey \\ 2 Kyoto Medical Center, National Hospital Organization, Clinical Research Institute, Kyoto, Japan \\ ${ }^{3}$ Atatürk University Faculty of Medicine, Department of Pediatrics, Erzurum, Turkey
}

\begin{abstract}
Hypoparathyroidism, deafness and renal dysplasia (HDR) syndrome is an autosomal dominant genetic disorder characterized by hypoparathyroidism, sensorineural deafness and renal dysplasia. We herein present the first Turkish patient with HDR syndrome, who has a p.R367X mutation. This report indicates that p.R367X is nota mutation specific for the Far Eastern populations and also that urological findings in infants with hypoparathyroidism should be carefully examined because clinical findings relating to the p.R367X mutation may show a variable age of onset.

Key words: HDR syndrome, infant, p.R367X mutation
\end{abstract}

Conflict of interest: None declared

Received: 12.01 .2015

Accepted: 10.03 .2015

\section{Introduction}

Hypoparathyroidism, deafness and renal dysplasia (HDR) syndrome is an autosomal dominant genetic disorder characterized by hypoparathyroidism, sensorineural deafness and renal dysplasia (1). Mutations in GATA3 gene located on the short arm of chromosome 10 are responsible for this syndrome. p.R367X is a mutation that causes a prematurely terminated protein with loss of basic amino acids in the flanking ZnF2 domain of GATA3 protein. To date, four HDR cases with heterozygous p.R367X mutation have been reported. Three of these cases were Japanese and Chinese $(2,3,4)$. We herein present the first Turkish patient genetically diagnosed with HDR syndrome, who has the same mutation. This report suggests that the p.R367X is not a mutation specific for the Far East populations and demonstrates that hypoparathyroidism and sensorineural deafness may display clinical variability, while the renal manifestation is limited to proteinuria and haematuria.

\section{Case Report}

A 2-month-old Turkish male infant, the second child of a nonconsanguineous marriage, was referred to our pediatric clinic with a history of an afebrile seizure. The patient was born at 40 gestational weeks by vaginal delivery after an unremarkable pregnancy. There was no family history of deafness or renal insufficiency. On admission, his vital signs were within normal limits. Weight, length and head circumference were $4470 \mathrm{~g}\left(25^{\text {th }}\right.$ percentile), $59 \mathrm{~cm}$ (50th percentile) and $39 \mathrm{~cm}$ (50th percentile), respectively. Physical examination findings were unremarkable. The low serum calcium (Ca) $6.4 \mathrm{mg} / \mathrm{dL}$; normal range, 8.8$10.3 \mathrm{mg} / \mathrm{dL})$, high serum phosphate $(7.2 \mathrm{mg} / \mathrm{dL}$; normal range, 3.8-6.5 $\mathrm{mg} / \mathrm{dL})$ and inappropriately low serum parathyroid hormone (PTH) (7 pg/mL; normal range, 20-65 pg/mL) levels 
of the patient led to a diagnosis of hypoparathyroidism. Serum alkaline phosphatase (ALP) and 25-hydroxy vitamin D [25(OH) D] levels were normal (388 U/L; normal range, 145-420 U/L and $22 \mathrm{ng} / \mathrm{mL}$; normal range, $20-75 \mathrm{ng} / \mathrm{mL}$, respectively). Urinalysis showed trace proteinuria and haematuria. Serum creatinine (Cr) level was high with $1.3 \mathrm{mg} / \mathrm{dL}$ (normal range, 0.2-0.4 $\mathrm{mg} / \mathrm{dL}$ ), while blood urea nitrogen was normal. Serum $\mathrm{Cr}$ level, proteinuria and haematuria persisted as $0.6-1.2 \mathrm{mg} / \mathrm{dL}$, trace/ $(1+)$ and trace $/(1+)$, respectively. Whole blood count, serum electrolytes including sodium, potassium and magnesium, liver tests and spot urine $\mathrm{Ca} / \mathrm{Cr}$ ratio were unremarkable. The thymus gland was present on the neck ultrasonography. Urinary ultrasonography, diethylenetriamine-pentaacetic acid, echocardiographyand auditory function tests were normal. Based on presence of hypoparathyroidism and renal findings, a diagnosis of HDR syndrome was made. The tetanic seizures were controlled by intravenous $\mathrm{Ca}$ gluconate, followed by treatment with elementary $\mathrm{Ca}(50 \mathrm{mg} / \mathrm{kg} /$ day $)$ and calcitriol $(0.25 \mu / d)$. The parents' serum Ca, P, ALP, PTH and 25(OH)D levels were 9.2 and $9.4 \mathrm{mg} / \mathrm{dL}, 3.7$ and $3.8 \mathrm{mg} / \mathrm{dL}, 323$ and 332 $\mathrm{U} / \mathrm{L}, 25$ and $35 \mathrm{pg} / \mathrm{mL}$ and 28 and $32 \mathrm{ng} / \mathrm{mL}$ in the mother and the father, respectively.

\section{Molecular Genetic Analysis}

Sequence analysis of coding and flanking intronic regions of the GATA3 gene was performed. The results showed a nonsense mutation ( $p$. R367X; c.1099C>T). The C>T mutation at nucleotide 1099 in exon 6 of the GATA3 resulted in substitution of a termination signal instead of arginine at codon 367. The patient's parents showed wild type sequences (Figure 1).

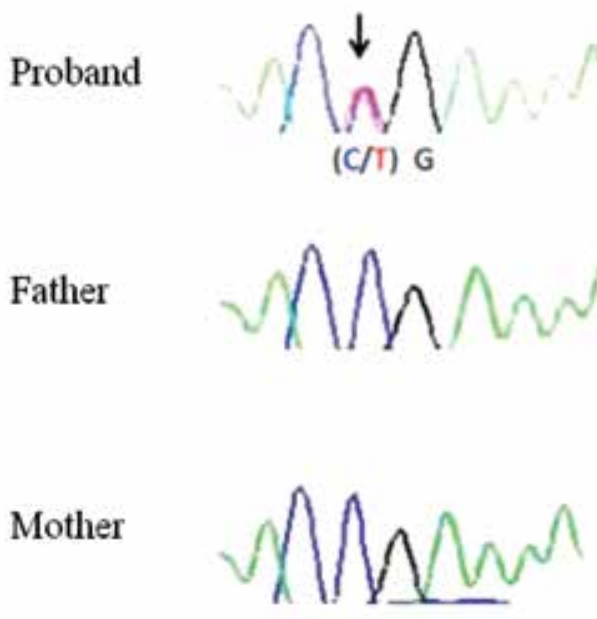

Figure 1. Electropherogram showing the heterozygous p.R367X mutation identified in the proband. The mutation was not detected in the mother's and father's samples. A black arrow points to the c. 1099C > T mutation which results in substitution of a termination signal instead of arginine at residue 367 (p.R367X)

\section{Discussion}

The human GATA3 gene is located on chromosome 10 p14 and consists of six exons (5). This gene encodes a 444-amino-acid transcription factor that contains two zinc finger [N-terminal (ZnF1) and C-terminal (ZnF2)] and two transactivating domains (6). ZnF2 domain is essential for DNA-binding, whereas ZnF1 domain stabilizes this binding and physically interacts with other multitype zinc finger proteins such as the Friends of GATA (FOG) (3). GATA3 mutations can be divided into three groups. The first group includes the mutations affecting the ZnF2 domain. They result in a loss of DNA-binding and represent about $90 \%$ of detected mutations. The second group is defined by a decrease of DNA-binding affinity due to the lack of $\mathrm{ZnF1}$ domain function. The third group have mutations characterized by normal DNA-binding and affinity but conformational changes of GATA3 protein or loss of interaction with FOG (7). The p.R367X mutation that we determined in our patient belongs to the first group and causes a prematurely terminated protein with loss of basic amino acids in the flanking ZnF2 domain. Four HDR cases with heterozygous p.R367X mutation have been reported to date (Table 1). Like our patient, no mutations were found in the reported patients' parents. This situation suggests that the mutation is de novo or results from a germinal mosaicism in either the father or the mother (4). Patients with p.R367 mutation have clinical variability in terms of age of onset as well as intensity and diversity of findings.

As observed in Table 1, cases 1, 3 and 4 were Japanese and Chinese. To the best of our knowledge, our patient is the first Turkish case genetically diagnosed to have HDR syndrome. This finding suggests that the p.R367X is not a mutation specific for the Far Eastern populations.

GATA3 gene is expressed in the central and peripheral nervous system, inner ear, thymus, parathyroid, liver, kidney and hematopoietic cell lineage (8). Parathyroid glands, inner ear and kidney are the most frequently affected organs due to GATA3 gene mutations. Hypoparathyroidism in patients with HDR syndrome displays the widest individual variability, ranging from asymptomatic hypocalcemia to paresthesias, muscular aching and a frank tetanic picture, with low, normal or even slightly elevated serum PTH levels (9). The age of onset is widely variable. Our case is the youngest patient who developed hypoparathyroidism among the reported cases with p.R367X mutation (Table 1).

The clinical feature of early-onset sensorineural deafness is the most completely penetrant aspect of the HDR syndrome. Diagnosis can be facilitated and anticipated by the observation of the variant hearing problem in a patient with hypoparathyroidism. It is unknown to what extent the deafness is caused by defects in the peripheral and/or central auditory systems. However, both types of defect may contribute to hearing loss because GATA3 gene is prominently expressed during development of 
Döneray $\mathrm{H}$ et al.

Hypoparathyroidism, Deafness and Renal Syndrome

Table 1. Clinical and laboratory characteristics of our patient and other reported cases with p.R367X mutation

\begin{tabular}{|c|c|c|c|c|c|}
\hline & Our patient & Case 1 (2) & Case 2 (3) & Case $3^{a}(4)$ & Case $4^{a}(4)$ \\
\hline Sex & Male & Female & Male & Male & Female \\
\hline Ethnic origin & Turkish & Japanese & No information & Chinese & Chinese \\
\hline Mutation in parents & No & No & No & No & No \\
\hline Presentation ${ }^{b}$ & $\mathrm{Se} / \mathrm{Te}$ & $\mathrm{Se} / \mathrm{Te}$ & $\mathrm{Se} / \mathrm{Te}$ & $\mathrm{Se} / \mathrm{Te}$ & $\mathrm{Se} / \mathrm{Te}$ \\
\hline Hypoparathyroidism & Yes & Yes & Yes & Yes & Yes \\
\hline Age at diagnosis & 2 months & 3 years & 13 years & 10 years & 5 years \\
\hline $\mathrm{Ca}(\mathrm{mg} / \mathrm{dL})^{\ddagger}$ & 6.4 & $8.1^{*}$ & $4.2^{*}$ & 6.5 & 8 \\
\hline$P(\mathrm{mg} / \mathrm{dL})^{\ddagger}$ & 7.2 & $3.4^{*}$ & No information & 9.1 & No information \\
\hline PTH $(\mathrm{pg} / \mathrm{mL})^{\ddagger}$ & 7 & No information & 6 & 1.6 & 3 \\
\hline Sensorineural deafness & No & Yes ${ }^{* *}$ & Yes & Yes** & Yes \\
\hline Age at diagnosis & -- & Probably childhood & $<1$ year & 3 years & 5 years \\
\hline Hearing level ${ }^{\dagger}(\mathrm{dB})$ & 10 & No information & No information & No information & No information \\
\hline Deafness symmetric & -- & No information & $\mathrm{B}, \mathrm{R}>\mathrm{L}$ & B & No information \\
\hline Renal abnormality & Yes & Yes & No & Yes & No \\
\hline Age at diagnosis & 2 months & Probably in the $2^{\text {nd }}$ decade & -- & 10 years & -- \\
\hline Findings & Mild proteinuria \& haematuria & Proteinuria \& haematuria & -- & Mild proteinuria & -- \\
\hline 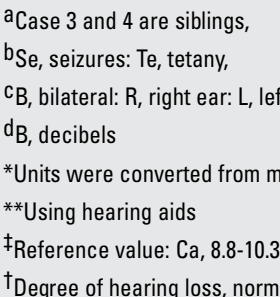 & L to $\mathrm{mg} / \mathrm{dL}$ & d $3.7-5.4 \mathrm{mg} / \mathrm{dL}$ in 4-15 year childr & PTH, 20-65 pg/mL & & \\
\hline
\end{tabular}

the ear as well that of the brain (10). The sensorineural deafness is usually bilateral, although the hearing loss may vary in its severity (1). The youngest case in terms of developing sensorineural deafness among the reported patients with p.R367X mutation was younger than 1 year. The onset in the other cases was during childhood. Cases 2 and 3 had bilateral sensorineural deafness and cases 1 and 3 were using hearing aids. Our patient showed no sensorineural deafness as yet. These findings suggest that the sensorineural deafness in patients with p.R367X mutation is variable in age of onset.

The renal phenotype of HDR syndrome is more variable. Renal manifestations may be uni-or bilateral and can include renal cysts, renal agenesis, renal dysplasia and vesico-ureteric reflux, variably represented even in affected members of the same family $(1,2)$. Patients with p.R367X mutation have no major renal abnormalities (Table 1). Cases 1 and 3 had proteinuria and haematuria, suggestive of renal dysplasia. Our patient is the youngest patient who developed renal manifestations among the reported cases. In addition to proteinuria and haematuria, he also had a high serum $\mathrm{Cr}$ level which persisted between 0.6 and $1.2 \mathrm{mg} / \mathrm{dL}$. The findings suggest that the p.R367X mutation does not cause major renal manifestations but lead to a high serum $\mathrm{Cr}$ level as well as to haematuria and proteinuria even in the infancy period.

In conclusion, we report the first Turkish case of a patient with HDR syndrome, who is also the youngest among the reported patients with $p . R 367 X$ mutation. This report indicates that the p.R367X mutation is not specific for the Far Eastern populations. p.R367X mutation demonstrates a variable age of onset of hypoparathyroidism, sensorineural deafness and renal abnormalities. This mutation is associated with proteinuria and haematuria but not major renal abnormalities. However, urological findings in infants with hypoparathyroidism should be carefully examined.

\section{References}

1. Bilous RW, Murty G, Parkinson DB, Thakker RV, Coulthard MG, Burn J, Mathias D, Kendall-Taylor P. Brief report: autosomal dominant familial hypoparathyroidism, sensorineural deafness, and renal dysplasia. N Engl J Med 1992;327:1069-1074. 
Döneray $\mathrm{H}$ et al.

Hypoparathyroidism, Deafness and Renal Syndrome

2. Muroya $K$, Hasegawa $T$, Ito $Y$, Nagai $T$, Isotani $H$, Iwata $Y$, Yamamoto K, Fujimoto S, Seishu S, Fukushima Y, Hasegawa Y, Ogata T. GATA3 abnormalities and the phenotypic spectrum of HDR syndrome. J Med Genet 2001;38:374-380.

3. Nesbit MA, Bowl MR, Harding B, Ali A, Ayala A, Crowe C, Dobbie A, Hampson G, Holdaway I, Levine MA, McWilliams $R$, Rigden $S$, Sampson J, Williams AJ,Thakker RV. Characterization of GATA3 mutations in the hypoparathyroidism, deafness, and renal dysplasia (HDR) syndrome. J Biol Chem 2004;279:22624-22634. Epub 2004 Feb 24

4. Sun $Y$, Xia $W$, Xing $X$, Li $M$, Wang $O$, Jiang $Y$, Pei $Y, Y e$ $P$, Liu $H$, Hu $Y$, Meng $X$, Zhou $X$. Germinal mosaicism of GATA3 in a family with HDR syndrome. Am J Med Genet A 2009;149A:776-778.

5. Van Esch $H$, Groenen $P_{1}$ Nesbit MA, Schuffenhauer $S$, Lichtner P, Vanderlinden G, Harding B, Beetz R, Bilous RW Holdaway I, Shaw NJ, Fryns JP, Van de Ven W, Thakker RV, Devriendt K. GATA3 haplo-insufficiency causes human HDR syndrome. Nature 2000;406:419-422.

6. Zahirieh A, Nesbit MA, Ali A, Wang $K$, He N, Stangou M, Bamichas G, Sombolos K, Thakker RV, Pei Y. Functional analysis of a novel GATA3 mutation in a family with the hypoparathyroidism, deafness, and renal dysplasia syndrome. J Clin Endocrinol Metab 2005;90:2445-2450. Epub 2005 Feb 10
7. Ali A, Christie PT, Grigorieva IV, Harding B, Van Esch $H$, Ahmed SF, Bitner-Glindzicz M, Blind E, Bloch C, Christin P, Clayton P, Gecz J, Gilbert-Dussardier B,Guillen-Navarro E, Hackett A, Halac I, Hendy GN, Lalloo F, Mache CJ, Mughal Z, Ong AC, Rinat C, Shaw N, Smithson SF, Tolmie J, Weill J, Nesbit MA, Thakker RV. Functional characterization of GATA3 mutations causing the hypoparathyroidism-deafness-renal (HDR) dysplasia syndrome: insight into mechanisms of DNA binding by the GATA3 transcription factor. Hum Mol Genet 2007;16:265-275. Epub 2007 Jan 8

8. Oosterwegel $M$, Timmerman J, Leiden J, Clevers $H$. Expression of GATA-3 during lymphocyte differentiation and mouse embryogenesis. Dev Immunol 1992;3:1-11.

9. Hernández AM, Villamar M, Roselló L, Moreno-Pelayo MA, Moreno F, Del Castillo I. Novel mutation in the gene encoding the GATA3 transcription factor in a Spanish familial case of hypoparathyroidism, deafness, and renal dysplasia (HDR) syndrome with female genital tract malformations. Am J Med Genet A 2007;143A:757-762.

10. Ferraris $S$, Del Monaco AG, Garelli E, Carando A, De Vito B, Pappi P, Lala R, Ponzone A. HDR syndrome: a novel "de novo" mutation in GATA3 gene. Am J Med Genet $A$ 2009;149A:770-775 